\title{
Surgical methods and efficacies for cervicothoracolumbar spinal schwannoma
}

\author{
QIANG DENG ${ }^{*}$, ZHENG TIAN*, WEIBIN SHENG, HAILONG GUO and MAI ER DAN \\ Department of Spinal Surgery, The First Affiliated Hospital of Xinjiang Medical University, \\ Urumqi, Xinjiang Uyghur Autonomous Region 830054, P.R. China
}

Received February 27, 2015; Accepted May 8, 2015

DOI: $10.3892 / e t m .2015 .2803$

\begin{abstract}
The aim of this study was to investigate the surgical methods and efficacies for cervicothoracolumbar spinal schwannoma (CSS). A total of 52 patients who had undergone treatment for schwannoma were retrospectively analyzed. Two methods were employed for the surgical resection of the thoracic and lumbar schwannomas: Type I (posterior midline approach semi-laminectomy with tumor resection and internal fixation with pedicle screws) was used in 24 cases, and type II (posterior midline approach laminectomy with tumor resection and internal fixation with pedicle screws) was used in 26 cases. Two cases of giant cervical schwannoma were treated via anterior-posterior combined surgery. Histopathological examination confirmed the diagnosis of schwannoma in all cases $(n=52)$. The clinical status of the patients was evaluated pre- and postoperatively using the visual analog scale, Oswestry Disability Index and Japanese Orthopedic Association scale, and the scores associated with the two types of surgical method were compared. Within the follow-up period, which lasted between 6 months and 3 years, no recurrence was detected, and such preoperative symptoms as radicular pain and spinal dysfunction were improved significantly. The numbness and hyperesthesia were relieved to different extents. In conclusion, methods of exposing and surgically treating CSS should be selected according to the growth site of the schwannoma in order to reduce the blood loss and surgery duration and to improve the surgical safety.
\end{abstract}

Correspondence to: Dr Weibin Sheng, Department of Spinal Surgery, The First Affiliated Hospital of Xinjiang Medical University, 137 Liyushan South Road, Urumqi, Xinjiang Uyghur Autonomous Region 830054, P.R. China

E-mail: weibinshengdoc@126.com

*Contributed equally

Key words: spine, schwannoma, surgical efficacy

\section{Introduction}

The schwannoma, also known as the Schwann cytoma, is the most common type of intraspinal tumor (1), accounting for approximately one-quarter of all primary intraspinal tumors. Due to the limited space in the spinal canal, the gradual growth of the schwannoma compresses the spinal cord and nerve roots, thus causing severe spinal and nerve root dysfunction. Early surgical treatment can result in a promising outcome (2-7).

To the best of our knowledge, few studies have systematically investigated cases of cervicothoracolumbar spinal schwannoma (CSS) (8); however, we propose that different surgical approaches should be applied according to different tumor growth sites, in order to improve the surgical safety. Pollo et al (8) and Jankowski et al (9) reported the use of an anterior-posterior combined surgical approach in the treatment of lumbar schwannoma. For thoracolumbar schwannomas, a suitable approach may be to perform a first-phase posterior tumor resection, i.e. posterior vertebral decompression plus intraspinal tumor resection, followed by extraspinal tumor resection. Canbay et al (10) suggested that spinal tumors measuring $>2.5 \mathrm{~cm}$ should be classified as giant tumors. Since intraoperative dissection of these tumors could damage the brachial plexus nerve and the adjacent vessels, thus generating complications, a suitable approach in such cases may be to first perform posterior vertebral decompression plus intraspinal tumor resection, followed by anterior tumor dissection and resection.

The aim of the present study was to investigate the surgical methods and efficacies for CSS. A total of 52 patients with CSS were surgically treated in the First Affiliated Hospital of Xinjiang Medical University (Urumqi, China) between 2011 and 2013, and their cases were retrospectively analyzed.

\section{Materials and methods}

General information. Fifty-two patients with intraspinal schwannoma were treated in the First Affiliated Hospital of Xinjiang Medical University between September 2011 and September 2013. The patients included 25 males and 27 females, who were aged 26-84 years (mean age, 47.5 years). Of the lesions, 2 were at the cervical spine, 23 were at the thoracic spine and 27 were at the lumbosacral spine, and radicular pain was the main clinical symptom. Twenty-six 
patients experienced radicular pain as the first symptom, which worsened when the patient slept at night or adopted a prostrate position and was alleviated when the patient moved or sat. A total of 27 patients exhibited numbness, weakness and hyperesthesia in the tumor-corresponding plane, and 2 patients exhibited painless scoliosis. This study was conducted in accordance with the Declaration of Helsinki and with approval from the Ethics Committee of Xinjiang Medical University. Written informed consent was obtained from all participants.

Auxiliary examination. All patients underwent normal spinal radiography, as well as spinal computed tomography (CT) and magnetic resonance imaging (MRI) examination of the corresponding sites. The general manifestations of X-ray and CT were an expanded intervertebral foramen and a widened spinal canal. MRI was considerably more effective at reflecting the status of the schwannoma: Extramedullary schwannomas, for example, were usually located at the dorsolateral side of the spinal cord, appearing class-round on the spinal surface. The Tl images showed iso- or slightly lower signals, while the T2 images showed iso- or slightly higher signals, with clear boundaries. The enhanced scanning showed homogeneous enhancement, and the cystic degeneration of the tumor was rare.

Surgical methods. In all patients, the surgery was performed under endotracheal intubation and intravenous general anesthesia with the patients in a prone position. The surgical methods for the thoracic and lumbar schwannomas were divided into types I and II according to the preoperative classification (11-14), and the patients were correspondingly assigned to groups 1 and 2 , respectively.

The type I method comprised a posterior midline approach semi-laminectomy with tumor resection and internal fixation with pedicle screws (Fig. 1), according to the preoperative diagnosis and analysis. The C-arm was used to position the lesion segment, and then used as the center to separate the subperiosteal paraspinal muscles, in order to expose the segmental spinous process affected by the tumor and the semi-lamina. The semi-laminectomy was performed towards the lesion side to resect the ipsilateral medial side of the facet process, in order to fully reveal the intervertebral foramen around the tumor. The longitudinal dura was subsequently incised along the para-tumor midline under the microscope and suspended bilaterally to expose the subdural space. When fully exposed, the boundaries of the tumor, nerves or spinal cord were clear, facilitating their identification. Normally, the arachnoid layer was closely attached to the tumor; this arachnoid layer exhibited a porous structure, independently surrounding the dorsal and ventral nerve roots. Subsequent to cutting this arachnoid layer, the tumor could be separated along the gaps between the tumor and pericoccygeal nerve, and the intraspinal part of tumor could then be resected. This was then tracked outwards along the affected vertebral foramen, and the section of the tumor between the vertebral foramen and the spinal canal could be gradually stripped and removed. If the exposure was limited, the operating table could be rotated towards the lesion side to facilitate the microscopic illumination and tumor exposure until the whole tumor was resected. Following complete resection, the dura was tightly sutured. The muscle or fascia tissues were used to cover the foramen and dural defects to prevent the leakage of cerebrospinal fluid. When lumbar tumors were found to be wrapped in coccygeal nerve or conus terminalis, the nerve roots were carefully separated to provide sufficient exposure to prevent recurrence.

The type II method comprised a posterior midline approach laminectomy with tumor resection and internal fixation with pedicle screws (Fig. 2). Monitored by a C-arm machine, the corresponding segments were subjected to pedicle screw implantation, laminectomy or lesion-ipsilateral laminectomy, lesion-ipsilateral facet resection and transverse process resection to reveal the whole tumor. Resection of the intraspinal section of the tumor was performed in an identical manner to that described in the type I method. The surgery was performed in the following sequence: Resection of the intraenvelope part, and separation and resection of the tumor envelope, in order to avoid damaging the coccygeal nerve or surrounding vital structures. If the dura was opened, it was sutured tightly. Following the resection of the tumor, the posterolateral spinal graft fusion and internal fixation of the pedicle screw were performed.

Postoperative treatment. Following surgery, antibiotics were routinely administered intravenously, prior to being withdrawn $48 \mathrm{~h}$ later. The patients with dural incision were additionally intravenously administered $80 \mathrm{mg}$ methylprednisolone and asked to adopt a supine position postoperatively, while patients with spinal meninges incision were requested to lie in the Trendelenburg position. Patients with internal fixation were able to perform ambulation 2 days later, while the patients without internal fixation could start functional exercise in bed 1 week later according to the situation. X-rays were carried out after 1 month to decide the ambulatory status according to the bone fusion conditions (15). Patients were asked to wear a conventional hard brace for 3 months of activities and to attend $\mathrm{X}$-ray and MRI appointments every 3 months. Follow-ups were performed for between 6 months and 3 years.

Evaluation of efficacies. The clinical status of each patient was evaluated pre- and postoperatively using the visual analog scale (VAS; $0 \mathrm{~mm}$, no pain; $100 \mathrm{~mm}$, worst imaginable pain), the Oswestry Disability Index (ODI) (16) and the Japanese Orthopedic Association (JOA) scores (17). Functional improvement was expressed by the recovery rate of the JOA scores. Pre- and postoperative neurological recovery was graded according to Frankel classification (18).

Statistical analysis. SPSS software (version 17.0; SPSS, Inc., Chicago, IL, USA) was used for the statistical analysis. The results are presented as the mean \pm standard deviation or range. The Student's t-test was used to perform the statistical comparisons. $\mathrm{P}<0.05$ was considered to indicate a statistically significant difference.

\section{Results}

Surgical methods. Thoracic and lumbar schwannomas were surgically resected using the aforementioned two methods (types I and II), and the patients were divided into two groups according to the different methods. In group 1 (posterior 

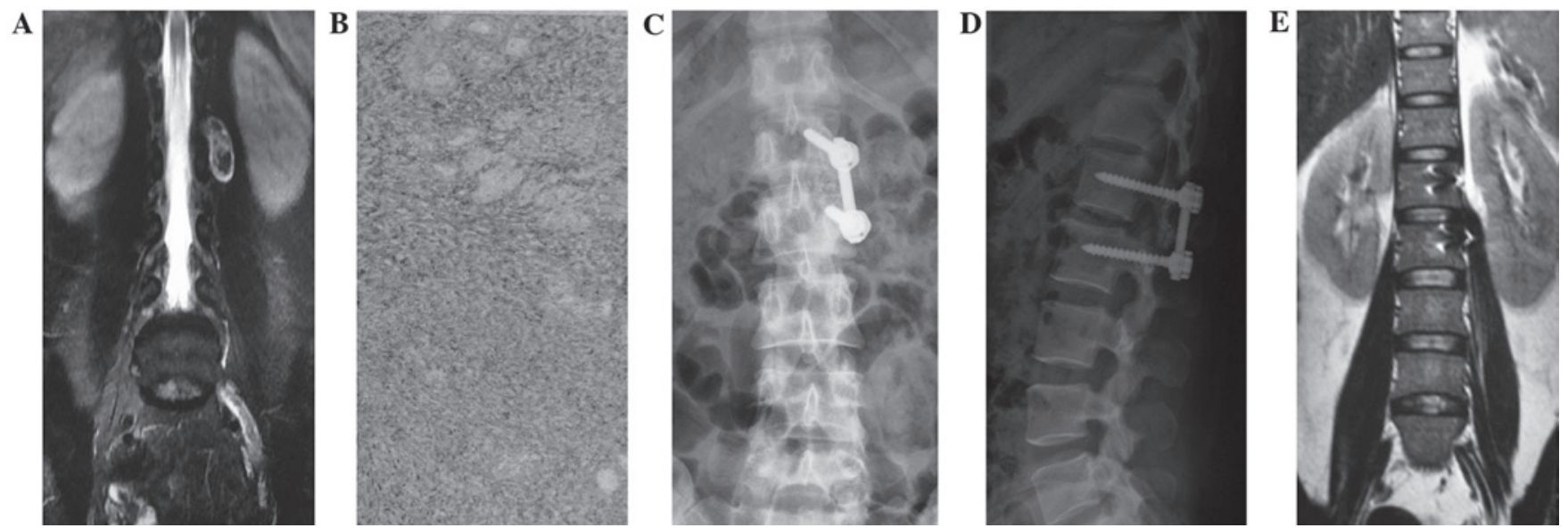

Figure 1. Posterior midline approach semi-laminectomy with tumor resection and internal fixation with pedicle screws. (A) Female, 34 years old: Lumbar 1/2 schwannoma. (B) Pathological report: Schwannoma. (C and D) Postoperative normal lumbar X-ray. (E) Magnetic resonance imaging 1 year after surgery revealed no recurrence.
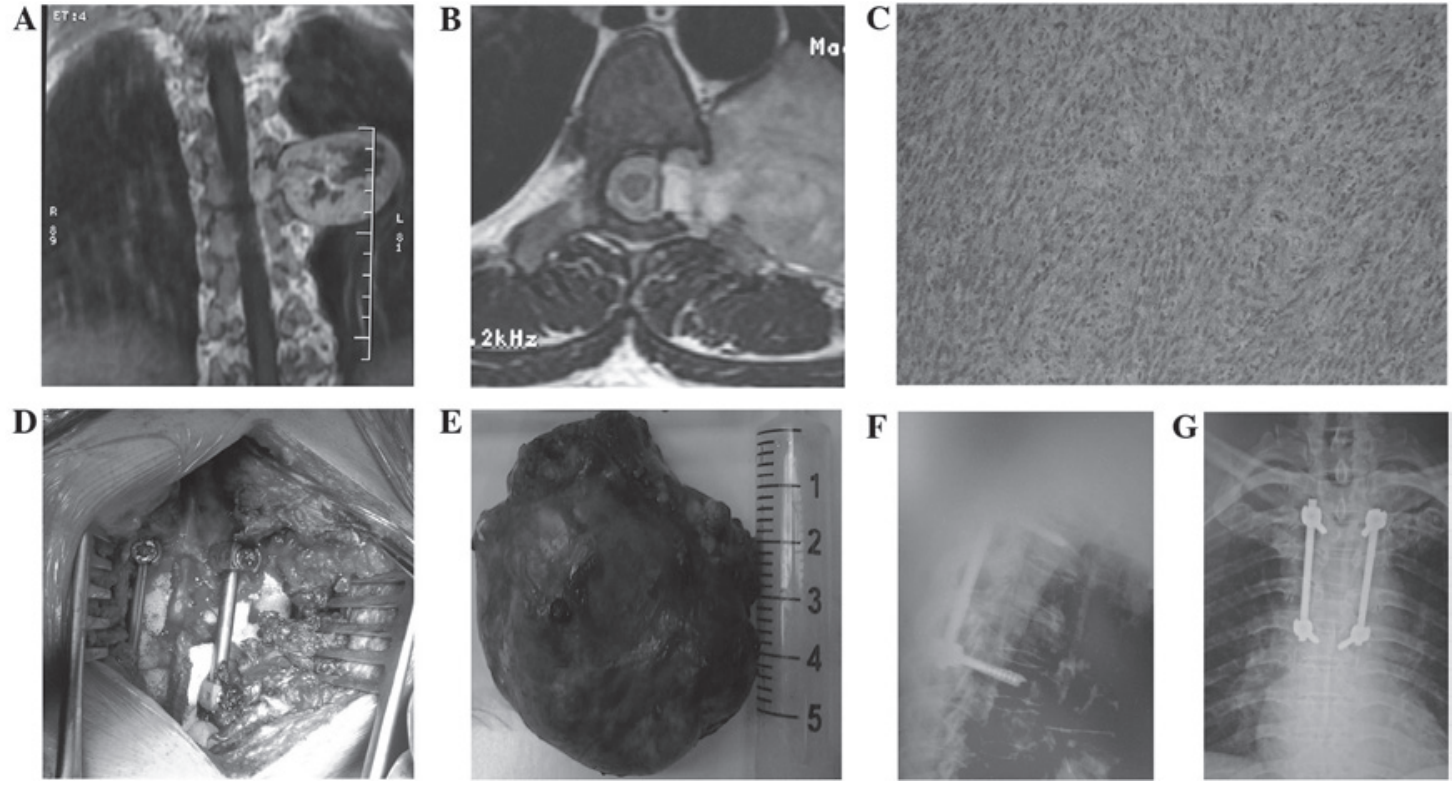

Figure 2. Posterior midline approach laminectomy with tumor resection and internal fixation with pedicle screws. (A and B) Male, 44 years old: Magnetic resonance imaging of a thoracic schwannoma. (C) Pathological report: Schwannoma. (D and E) Intraoperative internal fixation and complete resection of the schwannoma. (F and G) Postoperative normal thoracic X-ray.

midline approach semi-laminectomy with tumor resection and internal fixation with pedicle screws), which included 24 cases, the intraoperative blood loss was $300 \pm 20 \mathrm{ml}$, the duration of surgery was $2.4 \pm 1.3 \mathrm{~h}$ and the length of hospitalization was $8.4 \pm 5.8$ days. In group 2 (posterior midline approach laminectomy with tumor resection and internal fixation with pedicle screws), which included 26 cases, the intraoperative blood loss was $561 \pm 210 \mathrm{ml}$, the duration of surgery was $2.8 \pm 1.8 \mathrm{~h}$ and the length of hospitalization was $10.4 \pm 5.8$ days $(\mathrm{P}<0.05)$, intraoperative blood loss was $561 \pm 210 \mathrm{ml}(\mathrm{P}<0.05)$, the duration of surgery was $2.8 \pm 1.8 \mathrm{~h}(\mathrm{P}<0.05)$ and the length of hospitalization was $10.4 \pm 5.8$ days $(\mathrm{P}<0.05)$. Significant differences were detected between the two groups in length of hospitalization, intraoperative blood loss and the duration of surgery. The postoperative follow-up time was between 6 months and 3 years. During follow-up, no patients reported an exacerbation of the condition, and significant improvements in the radicular pain and spinal function were noted. The numbness and hyperesthesia were relieved to different extents, and the postoperative MRI revealed no recurrence during the 6-month to 3-year follow-up.

Pre-and postoperative clinical status. Prior to surgery, the mean VAS score was 31.5 in group 1 and 43.1 in group 2. Three months after surgery, the score decreased to $16.3(\mathrm{P}<0.001)$ in group 1 and $17.0(\mathrm{P}<0.001)$ in group 2, and at the final follow-up, the score had improved further to $12.1(\mathrm{P}<0.001)$ in group 1 and $12.4(\mathrm{P}<0.001)$ in group 2 (Table I). For each postoperative time-point, the difference between the two groups was insignificant. The mean preoperative ODI score in group 1 was 49.2, compared with $22.4(\mathrm{P}<0.001)$ at the 3 -month follow-up and $15.2(\mathrm{P}<0.001)$ at the final follow-up. The mean preoperative 
Table I. VAS, ODI and JOA scores of the two groups.

\begin{tabular}{lccc}
\hline Score & Group 1 & Group 2 & P-value \\
\hline VAS & & & $<0.05$ \\
Pre-operative & $31.5 \pm 14.1$ & $43.1 \pm 13.9$ & $\mathrm{NS}$ \\
3 months postoperative & $16.3 \pm 9.2$ & $17.0 \pm 10.3$ & $\mathrm{NS}$ \\
Final follow-up & $12.1 \pm 7.4$ & $12.4 \pm 7.6$ & $<0.05$ \\
ODI & & & $\mathrm{NS}$ \\
Pre-operative & $49.2 \pm 15.2$ & $62.8 \pm 11.8$ & $\mathrm{NS}$ \\
3 months postoperative & $22.4 \pm 9.3$ & $23.8 \pm 9.1$ & $<0.05$ \\
Final follow-up & $15.2 \pm 9.3$ & $16.9 \pm 9.5$ & $\mathrm{NS}$ \\
JOA & & $9.4 \pm 3.0$ & $\mathrm{NS}$ \\
Pre-operative & $14.4 \pm 2.0$ & $18.2 \pm 3.1$ & $21.6 \pm 2.4$ \\
3 months postoperative & $19.6 \pm 3.3$ & $22.7 \pm 3.2$ & \\
Final follow-up & & \\
\hline
\end{tabular}

Values are presented as the mean \pm standard deviation. NS, not significant; VAS, visual analog scale; ODI, Oswestry Disability Index; JOA, Japanese Orthopedic Association.

Table II. Improvements in the postoperative Frankel classification.

Postoperative Frankel classification

\begin{tabular}{lccccrr}
\cline { 2 - 6 } Preoperative Frankel classification & Cases (n) & A (n) & B (n) & C (n) & D (n) & E (n) \\
\hline A & 0 & 0 & 0 & 0 & 0 & 0 \\
B & 9 & 0 & 0 & 1 & 3 & 5 \\
C & 16 & 0 & 0 & 0 & 3 & 13 \\
D & 15 & 0 & 0 & 0 & 0 & 15 \\
E & 12 & 0 & 0 & 0 & 0 & 12 \\
\hline
\end{tabular}

ODI score in group 2 was 62.8, compared with $23.8(\mathrm{P}<0.001)$ at 3 months postoperatively and $16.9(\mathrm{P}<0.001)$ at the final follow-up (Table I). At each follow-up time-point, no significant difference was found between the two groups. The JOA scores for groups 1 and 2 were as follows: 14.4 and 9.4 prior to surgery, 19.6 and 18.2 at 3 months after surgery and 22.7 and 21.6 at the final follow-up, respectively (Table I). No significant difference was found between the two groups postoperatively.

At the final follow-up, 45 patients $(86.5 \%)$ considered their outcome to be excellent or good, 6 patients fair and 1 patient unchanged. The difference between the two groups was not statistically significant at the final follow-up. Frankel classification of neural function prior to surgery was as follows: Frankel B, 9 patients; Frankel C, 16 patients; Frankel D, 15 patients; and Frankel E, 12 patients. Neural function after follow-up was as follows: Frankel C, 1 patient; Frankel D, 6 patients; and Frankel E, 45 patients. The results for the postoperative Frankel classification are shown in Table II.

\section{Discussion}

Due to the biological characteristics of schwannomas, such as expansive growth, the surrounding important blood vessels, organs and spinal motor nerve roots can be dislodged; thus, surgical methods for the resection of the tumor capsule and the exposure and resection of the tumor should be selected to avoid damage to the surrounding organs and nerves (19-21). The posterior resection of the thoracic and lumbar schwannomas in the present study was found to be relatively safe, and the intraoperative internal fixation maximally retained the spinal biological stability; consequently, the patients rehabilitated more rapidly, and the duration of surgery and the length of hospitalization were shortened. Postoperative cerebrospinal fluid leakage occurred in 13 patients in this study, but this was cured through lumbar subarachnoid catheterization.

The thoracic and lumbar schwannomas in this study were surgically resected using two methods: The type I method was used in 24 cases and the type II method was used in 26 cases. Although patients in group 1 had a shorter duration of surgery and less intraoperative blood loss, the cases in group 2 involved a larger intraspinal tumor mass; therefore, the tumor tissues in the group 2 cases had to be fully exposed in order avoid damage to the spinal cord and nerve roots and prevent tumor residues. The surgical approach should be preoperatively selected according to the positions of the tumor and spinal nerve roots, so that surgical field exposure can be ensured. During thoracic 

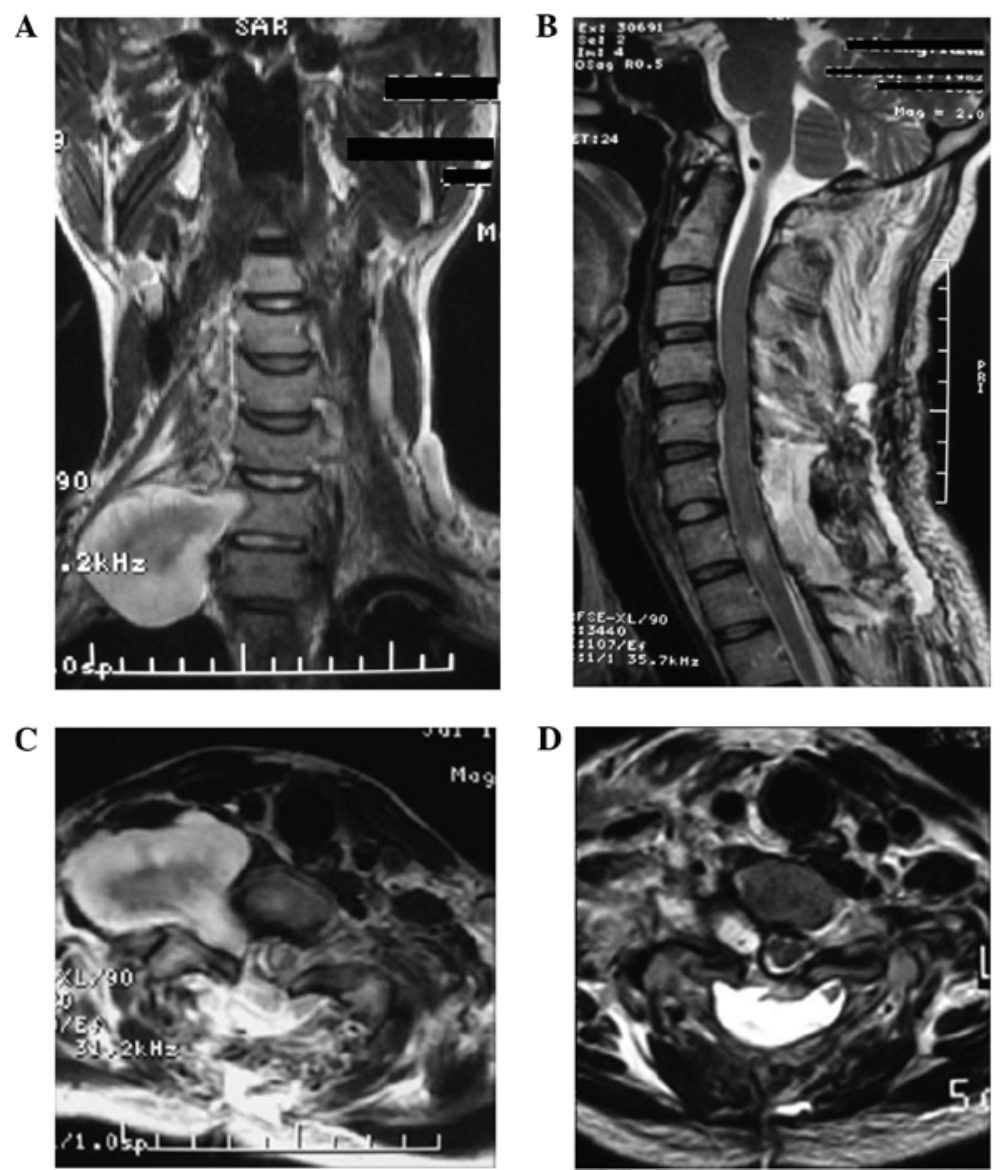

Figure 3. Posterior cervical laminar decompression and intraspinal tumor resection, followed by tumor dissection and resection through an anterior approach. (A) Female, 39 years old: MRI of the preoperative C7 schwannoma (coronal plane). (B) C6 and 7 posterior laminar decompression, partial resection of the schwannoma and partial resection of the affected right C7 nerve root (sagittal plane). (C) C7 posterior laminar decompression, partial resection of the schwannoma and partial resection of the affected right C7 nerve root (transverse plane). (D) Postoperative MRI: Phase II dumbbell-shaped giant schwannoma body resection through an anterior approach. MRI, magnetic resonance imaging (transverse plane).

schwannoma resection, the transverse process and ribs can be resected if necessary, and the maintenance of long-term stability should be ensured through precise intraoperative internal fixation and bone grafting. In total, 50 patients with thoracic schwannoma in this study underwent internal fixation, and the follow-up found no occurrence of spinal instability.

By analyzing the cases in the present study we found that methods I and II could both be used to completely resect CSSs. It was often difficult to reach larger schwannomas beside the cervical vertebra through the posterior approach, due to the abundance of the anterior cervical neurovascular structures, such as the brachial plexus nerves. In this study, 2 cases of dumbbell-shaped giant cervical schwannoma was resected using an anterior-posterior combined approach. First, C6 and 7 posterior laminar decompression was performed, followed by partial resection of the schwannoma and the affected right $\mathrm{C} 7$ nerve root. The dumbbell-shaped giant schwannoma body was then resected through the anterior approach, due to the adherence of the tumor to the upper and lower trunks of the brachial plexus nerve. The right brachial plexus nerve function gradually returned to preoperative levels 2 days later. Since only the cervical 6 and 7 laminae were resected, the cervical stability was affected minimally; therefore, internal fixation was not performed (Fig. 3).
Most schwannomas are located on the dorsal or lateral side of the spinal cord, and can be easily viewed when the dura is opened through the posterior approach. The tumors can thus be clearly intraoperatively separated, disconnected and isolated, and the capsule wall surface can be subjected to electric coagulation to shrink the tumor volume. The proximal and distal nerve roots of the tumor should be cut off to enable the removal of the whole tumor. For giant tumors, the intracapsular resection should be performed first to realize the intracapsular decompression, and the nerve roots of tumor origin should be cut off. The majority of dumbbell-shaped thoracolumbar tumors can be exposed by expanding the intervertebral foramen, and the whole facet resection of one side can increase the paraspinal exposure, thus enabling access to any extension of the tumor through the intervertebral foramen. Using this technique, all dissections can be performed on the tumor surface, the nerves can be identified from the proximal end and the nerve damage can be further reduced. Preserving the nerve roots at the expense of failing to perform a thorough tumor resection can lead to a higher local recurrence rate and a correspondingly increased necessity for further surgery (22).

Findings regarding the consequence of retaining or resecting the nerve roots affected by the tumor are not currently consistent $(22,23)$. In the majority of cases in which the nerve 
roots were resected, no permanent or serious dysfunction occurred, although a few cases exhibited slight sensory disturbances (22). In the present study, 52 affected nerve roots were resected without any obvious aggravation of sensory impairment. A possible explanation for the finding of this study is that the affected nerve roots gradually became deactivated with the long-term disease duration, leading to the adjacent spinal nerve roots compensatorily covering the surface area that was previously dominated by the affected nerve roots. We therefore propose that resecting the necessary affected nerve roots is likely to cause only mild sensory loss. In the 52 patients in the present study, the affected nerve roots were resected according to the situation; only 3 patients experienced skin numbness in the corresponding segmental areas following resection, and the skin numbness was improved significantly 1 year later. No permanent sensory impairments were caused.

The 52 patients in this study achieved satisfactory results following surgery; therefore, we believe that a detailed preoperative evaluation based on the preoperative MRI could be used to select the appropriate approach for surgery. This selection of the optimal surgical method could facilitate the complete removal of the tumor while causing a reduction in trauma.

\section{References}

1. Gottfried ON, Binning MJ and Schmidt MH: Surgical approaches to spinal schwannomas. Contemp Neurosurg 27: 1-9, 2005.

2. Lee SE, Chung CK and Kim HJ: Intramedullary schwannomas: Long-term outcomes of ten operated cases. J Neurooncol 113 : 75-81, 2013.

3. Colosimo C, Cerase A, Denaro L, Maira G and Greco R: Magnetic resonance imaging of intramedullary spinal cold schwannomas: Report of two case and review of the literature. J Neurosurg 99 (1 Suppl): 114-117, 2003.

4. Nakamura M, Iwanami A, Tsuji O, Hosogane N, Watanabe K, Tsuji T, Ishii K, Toyama $\mathrm{Y}$, Chiba $\mathrm{K}$ and Matsumoto $\mathrm{M}$ : Long-term surgical outcomes of cervical dumbbell neurinomas. J Orthop Sci 18: 8-13, 2013.

5. Matsuoka H, Itoh $\mathrm{Y}$, Numazawa $\mathrm{S}$, Tomii $\mathrm{M}$, Watanabe $\mathrm{K}$, Hirano Y and Nakagawa H: Recapping hemilaminoplasty for spinal surgical disorders using ultrasonic bone curette. Surg Neurol Int 3: 70, 2012.

6. Wu D, Ba Z, Huang Y, Zhao W, Shen B and Kan H: Totally cystic schwannoma of the lumbar spine. Orthopedics 36: e679-e682, 2013

7. Ozawa H, Kokubun S, Aizawa T, Hoshikawa T and Kawahara C: Spinal dumbbell tumors: An analysis of a series of 118 cases. J Neurosurg Spine 7: 587-593, 2007.
8. Pollo C, Richard A and De Preux J: Resection of a retroperitoneal schwannoma by a combined approach. Neurochirurgie 50: 53-56, 2004 (In French).

9. Jankowski R, Szmeja J, Nowak S, Sokół B and Blok T: Giant schwannoma of the lumbar spine. A case report. Neurol Neurochir Pol 44: 91-95, 2010.

10. Canbay S, Hasturk AE, Basmaci M, Erten F and Harman F: Management of thoracal and lumbar schwannomas using a unilateral approach without instability: An analysis of 15 cases. Asian Spine J 6: 43-49, 2012.

11. Wiedemayer H, Sandalcioglu IE, Aalders M, Wiedemayer H, Floerke M and Stolke D: Reconstruction of the laminar roof with miniplates for a posterior approach in intraspinal surgery: Technical consideration and critical evaluation of follow-up results. Spine (Phila Pa 1976) 29: E333-E342, 2004

12. McGirt MJ, Garcés-Ambrossi GL, Parker SL, Sciubba DM, Bydon A, Wolinksy JP, Gokaslan ZL, Jallo G and Witham TF: Short-term progressive spinal deformity following laminoplasty versus laminectomy for resection of intradural spinal tumors: Analysis of 238 patients. Neurosurgery 66: 1005-1012, 2010.

13. Yimaz MR, Bek S, Bekmezci T, Gökduman C and Solak AS: Malignant triton tumor of the lumbar spine. Spine (Phila $\mathrm{Pa}$ 1976) 29: E399-E401, 2004.

14. Fletcher CDM, Bridge JA, Hogendoorn PCW and Mertens F (eds.): World Health Organization Classification of Tumours of Soft Tissue and Bone. 4th edition. IARC Press, Lyon, 2013.

15. Theodosopoulos T, Stafyla VK, Tsiantoula P, Yiallourou A Marinis A, Kondi-Pafitis A, Chatziioannou A, Boviatsis E and Voros D: Special problems encountering surgical management of large retroperitoneal schwannomas. World J Surg Oncol 6: 107, 2008.

16. Fairbank JC, Couper J, Davies JB. The Oswestry Low Back Pain Questionnaire. Physiotherapy 1980; 66: 271-273.

17. Izumida, S, Inoue, S (1986) Assessment of treatment for low back pain. J Jpn Orthop Assoc 60: pp. 391-394.

18. Frankel HL, Hancock DO, Hyslop G, Melzak J, Michaelis LS, Ungar GH, Vernon JD and Walsh JJ: The value of postural reduction in the initial management of closed injuries of the spine with paraplegia and tetraplegia. I. Paraplegia 7: 179-192, 1969.

19. Ravnik J, Potrc S, Kavalar R, Ravnik M, Zakotnik B and Bunc G: Dumbbell synovial sarcoma of the thoracolumbar spine: A case report. Spine (Phila Pa 1976) 34: E363-E366, 2009.

20. Yokoi H, Arakawa A, Inoshita A and Ikeda K: Novel use of a Weerda laryngoscope for transoral excision of a cervical ganglioneuroma: A case report. J Med Case Rep 6: 88, 2012.

21. Celii P, Trillò G and Ferrante L: Spinal extradural schwannoma. J Neurosurg Spine 2: 447-456, 2005.

22. Schulthiess R and Gullotta G: Resection of relevant nerve roots in surgery of spinal neurinomas without persisting neurological deficit. Acta Neurochir (Wien) 122: 91-96, 1993.

23. Seppälä MT, Hatia MJ, Sankila RJ, Jääskeläinen JE and Heiskanen O: Long-term outcome after removal of spine schwannoma: A clinicopathological study of 187 cases. J Neurosurg 83: 621-626, 1995. 